\title{
SOLUCIÓN LOCAL PARA UNA CLASE DE ECUACIONES NO-LINEALES DE TIPO KIRCHHOFF
}

\author{
Raúl Izaguirre Maguiña \\ Universidad Nacional Mayor de San Marcos
}

\section{RESUMEN}

Sea A el operador definido por la terna $\{H, V ;(())$,$\} donde H, V$ son espacios de Hilbert, la inmersión de $V$ en $H$ es densa y compacta. Consideramos el problema:

$$
\mid \begin{aligned}
& u^{\prime \prime}(t)+M\left(\left|A^{\alpha} u(t)\right|^{2}\right) A^{\beta} u(t)=f(t) \\
& u(0)=u_{0}, u^{\prime}(0)=u_{1} .
\end{aligned}
$$

Se prueba la existencia de solución local para la ecuación tipo Kirchhoff $\left(^{*}\right)$, para todo $\alpha, \beta$ números reales tales que $\alpha>0 y \beta>0$.

\section{INTRODUCCIÓN}

Sea $\Omega$ un abierto de $\Re^{n}$ con frontera regular; $Q$ el cilindro $\Omega x[0, T], 0<T<\infty$, con frontera lateral $\Sigma$. La siguiente ecuación diferencial parcial no-lineal,

$$
\begin{array}{ll}
u_{t t}-M\left(\int_{\Omega} \mid \nabla u(x, t)^{2} d x\right) \Delta u=f & \text { en } Q \\
u=0 & \text { en } \Sigma \\
u(x, 0)=u_{0}(x), u^{\prime}(x, 0)=u_{1}(x) & \text { en } \Omega
\end{array}
$$

es conocida como la ecuación casi lineal de Kirchhoff, que tiene su motivación física en el estudio de las vibraciones de pequeña amplitud de una cuerda fija en sus extremos y cuando la dependencia de la tensión no puede dejarse de lado en el modelo. 
El caso $M(s)=s$, fué propuesto por Lions [9]. Resultados sobre el problema de Kirchhoff para diferentes condiciones para la función $\mathrm{M}$, el abierto $\Omega$, la dimensión n y los datos iniciales, pueden encontrarse por ejemplo, en las referencias [3], [4], [12], [11], [9]. En particular, en la referencia [9], J.L. Lions estudia el problema de Kirchhoff en un contexto mas amplio y abstracto, planteando la ecuación:

$$
u^{\prime \prime}(t)+M\left(\mid A^{1 / 2} u(t)\right) A u(t)=f(t)
$$

donde A representa un operador auto-adjunto, positivo de un espacio de Hilbert $H$ y $A^{1 / 2}$ representa su raíz cuadrada.

Para el caso de la ecuación $(* *)$, se tienen las referencias [1], [9], [5], [13]. En particular en [11], L.A. Medeiros-M.Milla Miranda demuestran que si $u_{0} \in D\left(A^{3 / 4}\right) u_{1} \in D\left(A^{1 / 4}\right) y \quad f \in L^{2}\left(0, T ; D\left(A^{1 / 4}\right)\right.$, entonces existe una solución local débil al problema de Cauchy asociado a (**).

En el presente trabajo estudiamos una clase de ecuaciones de tipo Kirchhoff de la forma :

$$
u^{\prime \prime}(t)+M\left(\left|A^{\alpha} u(t)\right|^{2}\right) A^{\beta} u(t)=f(t)
$$

que incluye como caso particular el problema estudiado en [9] $(\alpha=1 / 2$ y $\beta=1)$.

\section{PRELIMINARES}

Sean $(V,(())) ;,(H,()$,$) espacios de Hilbert, la inmersión de \mathrm{V}$ en $\mathrm{H}$ densa y compacta. Sea también A el operador definido por la terna $\{V, H ;(()))\}$.

Entonces, $D(A)$ es un subespacio denso en $H, A$ es un operador no-acotado autoadjunto y positivo de $H$, con espectro discreto;

$$
A w_{v}=\lambda_{v} w_{v} ; \lambda_{v}>0, \forall v ; y \lambda_{v} \rightarrow \infty \quad \text { cuando } \quad v \rightarrow \infty
$$


$\left\{w_{v}\right\}$ es un sistema ortonormal completo de $H$ de modo que:

$$
\begin{aligned}
& D(A)=\left\{u \in H\left|\sum_{v=1}^{\infty} \lambda_{v}^{2}\right|\left(u, w_{v}\right)^{2}<\infty\right\} \\
& A u=\sum_{v=1}^{\infty} \lambda_{v}\left(u, w_{v}\right) w_{v}, \quad \forall u \in D(A) .
\end{aligned}
$$

Asímismo para todo $\alpha>0$, el operador $A^{\alpha}$ está bien definido

$$
\begin{aligned}
& D\left(A^{\alpha}\right)=\left\{u \in H\left|\sum_{v=1}^{\infty} \lambda_{v}^{2 \alpha}\right|\left(u, w_{v}\right)^{2}<\infty\right\} \\
& A^{\alpha} u=\sum_{v=1}^{\infty} \lambda_{v}^{\alpha}\left(u, w_{v}\right) w_{v}, \quad \forall u \in D\left(A^{\alpha}\right) .
\end{aligned}
$$

Haciendo:

$$
\|u\|_{\alpha}^{2}=|u|^{2}+\left.A^{\alpha} u\right|^{2}
$$

se tiene que $\left(D\left(A^{\alpha}\right), \|_{\alpha}\right)$ es un espacio de Hilbert y que la dimensión de $D\left(A^{\alpha}\right)$ en $H$ es compacta.

Si $\beta>\alpha \geq 0$, entonces la inmersión de $D\left(A^{\beta}\right)$ en $D\left(A^{\alpha}\right)$ es también compacta. Además se tiene que los operadores $A^{-\alpha}$ son compactos con $D\left(A^{-\alpha}\right)=H$ y definidos por:

$$
A^{-\alpha} u=\sum \lambda_{v}^{-\alpha}\left(u, w_{v}\right) w_{v} \quad \forall u \in H
$$

En todo el trabajo, la función M, verifica las siguientes condiciones:

$$
\begin{aligned}
& M_{1}: \quad M \in C^{1}([0, \infty[) \\
& M_{2}: \quad M(s) \geq s_{0}>0, \quad \forall s>0 .
\end{aligned}
$$


Usamos la notación

$$
\hat{M}(s)=\int_{0}^{s} M(\tau) d \tau
$$

\section{TEOREMA DE EXISTENCIA 1}

Sea $\alpha>\beta / 2, u_{0} \in\left(D\left(A^{n}\right)\right), u_{1} \in\left(D\left(A^{\varepsilon}\right)\right), f \in L^{2}\left(0, T ; D\left(A^{n}\right)\right)$

donde:

$$
n=\operatorname{Max}\{(4 \alpha+\beta) / 4, \beta / 2\} \quad y \quad \varepsilon=(4 \alpha-\beta) / 4,
$$

entonces existe un $T_{0}$ tal que $0<T_{0}<T$ y una función vectorial $u:[0, T] \rightarrow D\left(A^{n}\right.$ satisfaciendo las siguientes condiciones:

$$
\begin{gathered}
u \in L^{\infty}\left(0, T ; D\left(A^{n}\right)\right. \\
u^{\prime} \in L^{\infty}\left(0, T ; D\left(A^{\varepsilon}\right)\right) \\
\frac{d}{d t}\left(u^{\prime}(t), v\right)+M\left(\mid A^{\alpha} u(t)^{2}\right)\left(A^{\beta / 2} u(t), A^{\beta / 2} v\right)=(f(t), v)
\end{gathered}
$$

en el sentido de $D^{\prime}\left(0, T_{0}\right)$, para todo $v \in D\left(A^{n}\right)$.

\section{Demostración:}

Sea $\left\{w_{v}\right\}$ el sistema ortonormal completo de $H$ formado por los vectores propios del operador $A$.

$$
\begin{aligned}
& V_{m}=\left[w_{1}, \ldots ., w_{m}\right] \\
& u_{m}(t)=\sum h_{i}(t) w_{i} \in V_{m},
\end{aligned}
$$


tal que:

$$
\begin{gathered}
\left(u_{m}^{\prime \prime}(t), v\right)+M\left(\left|A^{\alpha} u_{m}(t)\right|^{2}\right)\left(A^{\beta} u_{m}(t), v\right)=(f(t), v), v \in V_{m} \\
u_{m}(0)=u_{0 m} \rightarrow u_{0} \text { en } D\left(A^{n}\right) \\
u_{m}^{\prime}(0)=u_{1 m} \rightarrow u_{1} \text { en } D\left(A^{\varepsilon}\right.
\end{gathered}
$$

ESTIMATIVA 1. Haciendo $v=2 A^{\gamma} \dot{U}_{m}^{\prime}(t)$ donde $\gamma=2 \alpha-\beta$, en el problema aproximado, obtenemos:

$$
\begin{aligned}
& \frac{d}{d t}\left\{\left|A^{\gamma / 2} u_{m}^{\prime}(t)\right|^{2}+\hat{M}\left(\left|A^{\alpha} u_{m}(t)\right|^{2}\right)\right\}=2\left(A^{\gamma / 2} f(t), A^{\gamma / 2} u_{m}^{\prime}(t)\right. \\
& \quad \leq\left|A^{\gamma / 2} f(t)\right|^{2}+\left|A^{\gamma / 2} u_{m}^{\prime}(t)\right|^{2} .
\end{aligned}
$$

Integrando de 0 a $t$.

$$
\begin{gathered}
\left|A^{\gamma / 2} u_{m}^{\prime}(t)\right|^{2}+\hat{M}\left(\left|A^{\alpha} u_{m}(t)\right|^{2}\right) \leq \int_{b}^{t}\left|A^{\gamma / 2} f(t)\right|^{2}+\int_{b}^{t} \mid A^{\gamma / 2} u_{m}^{\prime}(t)^{2}+ \\
\left|A^{\gamma / 2} u_{l m}\right|^{2}+M\left(\left|A^{\alpha} u_{0 m}\right|^{2}\right) \leq C .
\end{gathered}
$$

Entonces, utilizando el lema de Gronwall y las convergencias en (5) y (6), obtenemos que:

$$
\left|A^{\gamma / 2} u_{m}^{\prime}(t)\right|^{2}+\hat{M}\left(\mid A^{\alpha} u_{m}(t)^{2}\right) \leq C .
$$


Donde $C$ ahora y en lo que sigue representa constantes que son independientes de $m$ y $t$. Luego por la condición $M_{2}$ se tiene que:

$$
u_{m} \text { está acotada en } L^{\infty}\left(0, T ; D\left(A^{\alpha}\right)\right)
$$

ESTIMATIVA 2. Haciendo $v=2 A^{2 \varepsilon} u_{m}^{\prime}(t)$ en el problema aproximado, obtenemos:

$$
\begin{aligned}
& \frac{d}{d t}\left\{\left|A^{\varepsilon} u_{m}^{\prime}(t)^{2}+M\left(\mid A^{\alpha} u_{m}(t)^{2}\right)\right| A^{(\beta+2 \varepsilon) / 2} u_{m}(t)^{2}\right\}=\left(A^{\alpha} f(t), A^{\varepsilon} u_{m}^{\prime}(t)\right)+ \\
& -2 M^{\prime}\left(\mid A^{\alpha} u_{m}(t)^{2}\right)\left(A^{\alpha} u_{m}^{\prime}(t)\right)\left|A^{(\beta+2 \varepsilon) / 2} u_{m}(t)^{2} \leq\right| A^{\varepsilon} f(t)^{2}+A^{\varepsilon} u_{m}^{\prime}(t)^{2}+ \\
& C A^{\varepsilon} u_{m}^{\prime}(t) \mid A^{(\beta+2 \varepsilon) / 2} u_{m}(t)^{2} .
\end{aligned}
$$

Sea:

$$
\varphi(t)=\left|A^{(4 \alpha-\beta) / 4} u_{m}^{\prime}(t)^{2}\right| A^{(4 \alpha+\beta) / 4} u_{m}(t)^{2}
$$

Integrando de 0 a $t$, teniendo en cuenta (5), (6) y la estimativa (8), obtenemos una desigualdad de la forma:

$$
\varphi(t) \leq c+c \int_{0}^{t} \varphi(s) d s+c \int_{0}^{t} \varphi^{2}(s) d s .
$$

Luego, existe $T_{0}>0$, tal que :

$$
\varphi(t) \leq c \quad \forall t \in\left[0, T_{0}\right] .
$$

Por tanto:

$$
\begin{aligned}
& \left(u_{m}\right) \text { está acotada en } L^{\infty}\left(0, T_{0} ; D\left(A^{(4 \alpha+\beta) / 4}\right)\right. \\
& \left(u_{m}^{\prime}\right) \text { está acotada en } L^{\infty}\left(0, T_{0} ; D\left(A^{(4 \alpha+\beta) / 4}\right)\right.
\end{aligned}
$$


ESTIMATIVA 3. Haciendo $v=2 u_{m}^{\prime}(t)$, en el problema aproximado obtenemos:

$$
\begin{aligned}
& \frac{d}{d t}\left\{\left|u_{m}^{\prime}(t)^{2}+M\left(\mid A^{\alpha} u_{m}(t)^{2}\right)\right| A^{\beta / 2} u_{m}(t)^{2}\right\}=\left(f(t), u_{m}^{\prime}(t)\right)+ \\
& \quad+M^{\prime}\left(\left|A^{\alpha} u_{m}(t)\right|^{2}\right)\left(A^{\alpha} u_{m}(t), A^{\alpha} u_{m}^{\prime}(t)\right)\left|A^{\beta / 2} u_{m}(t)\right|^{2} \leq \mid f(t)^{2} \\
& \quad+\left|u_{m}^{\prime}(t)^{2}+c\right| A^{\beta / 2} u_{m}(t)^{2} .
\end{aligned}
$$

donde hemos tenido en cuenta que:

$$
\mid\left(A^{\alpha} u_{m}(t), A^{\alpha} u_{m}^{\prime}(t)|\leq| A^{(4 \alpha-\beta) / 4} u_{m}^{\prime}(t) \mid \leq c\right.
$$

Sea:

$$
\varphi_{1}(t)=\left|u_{m}^{\prime}(t)\right|^{2}+\left|A^{\beta / 2} u_{m}(t)\right|^{2}
$$

Entonces, integrando de 0 a t, teniendo en cuenta (5), (6) y las estimativas (9) y (10), obtenemos una desigualdad de la forma:

$$
\varphi_{1}(t) \leq c+c \int_{0}^{t} \varphi_{1}(s) d s
$$

Por el lema de Gronwall, obtenemos:

$$
\varphi_{1}(t)=\left|u_{m}^{\prime}(t)\right|^{2}+\mid A^{\beta / 2} u_{m}(t)^{2} \leq c \quad \forall t \in\left[0, T_{0}\right]
$$

Luego

$$
\begin{aligned}
& \left(u_{m}\right) \text { está acotada en } L^{\infty}\left(0, T_{0} ; D\left(A^{\beta / 2}\right)\right. \\
& \left(u_{m}^{\prime}\right) \text { está acotada en } L^{\infty}\left(0, T_{0} ; H\right)
\end{aligned}
$$

Con las estimativas obtenidas y procediendo como en la referencia [11], se deduce que existe una función $u$ que verifica las condiciones del teorema 1. 


\section{TEOREMA DE EXISTENCIA 2}

Sea $0 \leq \alpha<\beta / 4, u_{0} \in D\left(A^{(2 \alpha+\beta) / 2}\right), \quad u_{1} \in D\left(A^{\alpha}\right), \quad f \in L^{2}\left(0, T ; D\left(A^{\alpha}\right)\right) . \quad$ Entonces existe un $T_{0}$, tal que $0<T_{0}<T$ y una función vectorial $u:[0, T] \rightarrow D\left(A^{\alpha}\right.$ satisfaciendo las siguientes condiciones:

$$
\begin{gathered}
u \in L^{\infty}\left(0, T ; D\left(A^{(2 \alpha+\beta) / 2}\right)\right) \\
u^{\prime \prime} \in L^{\infty}\left(0, T ; D\left(A^{\alpha}\right)\right) \\
\frac{d}{d t}\left(u^{\prime}(t), v\right)+M\left(\mid A^{\alpha} u(t)^{2}\right)\left(A^{\beta / 2} v\right)=(f(t), v)
\end{gathered}
$$

en el sentido de $D^{\prime}\left(0, T_{0}\right)$, para todo $v \in D\left(A^{\alpha}\right)$.

\section{Demostración :}

Como en el teorema 1 , sea $\left\{w_{v}\right\}$ el sistema ortonormal completo de $H$, formado por los vectores propios del operador $A$.

$$
\begin{aligned}
& V_{m}=\left[w_{1}, \ldots ., w_{m}\right] \\
& u_{m}(t)=\Sigma h_{i}(t) w_{i} \in V_{m},
\end{aligned}
$$

tal que:

$$
\begin{aligned}
& \left(u_{m}^{\prime \prime}(t), v\right)+M\left(A^{\alpha} u_{m}(t)\right)\left(A^{\beta} u_{m}(t), v\right)=(f(t), v), v \in V_{m} \\
& u_{m}(0)=u_{0 m} \rightarrow u_{0} \quad \text { en } \quad D\left(A^{(2 \alpha+\beta) / 2}\right. \\
& u_{m}^{\prime}(0)=u_{1 m} \rightarrow u_{1} \quad \text { en } \quad D\left(A^{\alpha}\right)
\end{aligned}
$$


ESTIMATIVA 1. Haciendo $v=2 A^{\gamma} u_{m}(t)$ donde $\gamma=2 \alpha-\beta$ en el problema aproximado, obtenemos:

$$
\begin{aligned}
\frac{d}{d t}\left\{\left|A^{\gamma / 2} u_{m}^{\prime}(t)\right|^{2}\right. & \left.+M\left(\left|A^{\alpha} u_{m}(t)\right|^{2}\right)\right\}=2\left(A^{\gamma / 2} f(t), A^{\gamma / 2} u_{m}^{\prime}(t)\right) \\
& \left.\leq\left. A^{\gamma / 2} f(t)\right|^{2}+A^{\gamma / 2} u_{m}^{\prime}(t)\right)^{2}
\end{aligned}
$$

Integrando de 0 a $t$

$$
\begin{aligned}
& \left|A^{\gamma / 2} u_{m}^{\prime}(t)\right|^{2}+M\left(\mid A^{\alpha} u_{m}(t)^{2}\right) \leq \int_{b}^{t}\left|A^{\gamma / 2} f(t)+\int_{b}^{t}\right| A^{\gamma / 2} u_{m}^{\prime}(t) \\
& +\left|A^{\gamma / 2} u_{I m}\right|^{2}+M\left(\left|A^{\alpha} u_{0 m}\right|^{2}\right)\left|A^{\alpha} u_{0 m}\right|^{2} \leq c .
\end{aligned}
$$

Por el lema de Gronwall y las convergencias (16), (17) obtenemos que:

$$
\left(u_{m}\right) \text { está acotada en } L^{\infty}\left(0, T_{0 ;} D\left(A^{\alpha}\right)\right.
$$

ESTIMATIVA 2. Haciendo $v=2 A^{2 \alpha} u_{m}^{\prime}(t)$, en el problema aproximado, obtenemos:

$$
\begin{aligned}
& \frac{d}{d t}\left\{\left.\left|A^{\varepsilon} u_{m}^{\prime}(t)^{2}+M\left(\mid A^{\alpha} u_{m}(t)^{2}\right)\right| A^{(2 \alpha+\beta)} u_{m}(t)\right|^{2} A^{(2 \alpha+\beta)} u_{m}(t)^{2}\right\}=2\left(A^{\alpha} f(t), A^{\varepsilon} u_{m}^{\prime}(t)\right)+ \\
& -M^{\prime}\left(\left|A^{\alpha} u_{m}(t)\right|^{2}\right)\left(A^{\alpha} u_{m}(t), A^{\alpha} u_{m}^{\prime}(t)\right) \mid A^{(2 \beta+\beta) / 2} u_{m}(t)^{2} \leq A^{\varepsilon} f(t)^{2}+A^{\varepsilon} u_{m}^{\prime}(t)^{2}+ \\
& \left.c\left|A^{\varepsilon} u_{m}^{\prime}(t)\right| A^{(2 \beta+\beta) / 2} u_{m}(t)\right|^{3} .
\end{aligned}
$$

Sea $\rho(t)=\left|A^{\alpha} u_{m}^{\prime}(t)\right|^{2}+\left|A^{(2 \alpha+\beta) / 2} u_{m}(t)\right|^{2}$.

Integrando de 0 a t, teniendo en cuenta las convergencias (16), (17) y la estimativa (18), obtenemos una desigualdad de la forma:

$$
\rho(t) \leq c+c \int_{0}^{1} \rho(s) d s+c \int_{0}^{1} \rho^{2}(s) d s .
$$


Luego, existe $T_{0}>0$, tal que:

$$
\rho(t) \leq c \quad \forall t \in\left[0, T_{0}\right] .
$$

Por tanto:

$$
\begin{aligned}
& \left(u_{m}\right) \text { está acotada en } L^{\infty}\left(0, T_{0} ; D\left(A^{2 \alpha+\beta / 2}\right)\right. \\
& \left(u_{m}^{\prime}\right) \text { está acotada en } L^{\infty}\left(0, T_{0} ; D\left(A^{\alpha}\right)\right)
\end{aligned}
$$

Con las estimativas obtenidas y procediendo como por ejemplo en la referencia [11], se deduce que existe una función $u$ que verifica las condiciones del teorema 2.

\section{REFERENCIAS}

1. A. AROSIO-S. SPAGNOLO. "Global solutions of the Cauchy problem for a non-linear Hyperbolic Equation". Universita di Pisa. Departamento de Matematica. Roma (1982).

2. N. ANDRADE. "On a non-linear system of partial differential equations". J. Math. Analysis Applic (1983).

3. R. DICKEY. "The initial value problem for a non-linear semi-infinite string". Proc. R. Soc. (1978).

4. R. DICKEY. "Infinite system of non-linear oscilations equations related to string". Proc. Amer. Math. Soc. Vol 23 (1969).

5. Y. EBIHARA. "On solutions of semilinear wave equation". Nonlinear Analysis, 6 (1982).

6. Y. EBIHARA-L. MEDEIROS-M. MILLA MIRANDA. "Local solutions for a nonlinear degenerate hyperbolic equations". Nonlinear Analysis, Vol. 10 (1986).

7. Y. EBIHARA. "On the existence of local smooth solutions for same degenerate quasilinear hyperbolic equations".Anais Acad. Bras. Ciencias, Vol. 57 (1985).

8. J. LIMACO FERRELL. "Existencia de soluçoes para a equaçao da corda elástica com amortecimiento". Atas do $37^{\circ}$ Seminário Brasilero de Análise.

9. J. L. LIONS. "Quelques Méthodes de Résolution des Problemes aux Limites Nonlinéare". Dunod, Paris (1969).

10. L. MEDEIROS-M. MILLA MIRANDA. "Remarks on a nonlinear model vibrations of string with damping". $30^{\circ}$ Seminario Brasilero de Análise, LNCC, RJ (1989). 
11. L. MEDEIROS-M . MILLA MIRANDA. "Local solutions for a nonlinear unilateral problem". Rev. Roumaine Math. App. Vol. 31 (1986).

12. N. LARKIN. "The unilateral problem for the local quasilinear hyperbolic equations of the theory of elasticity". Soviet. Physics Docklay. Vol. 29 (1984).

13. G.P. MENZALA. "On classical solutions of a quasilinear hyperbolic equations". Nonlinear Analysis, Vol. 3 (1979).

14. S. POHOZAEV. "The Kirchhoff quasilinear hyperbolic equations". Differential Equations, Vol. 21 (1985).

15. P.H. RIVERA. "On local strong solutions of a nonlinear partial differential equation". Appl. Analysis, Vol. $10(1980)$. 